\title{
THE X-RAY PROPERTIES OF NEARBY ABELL CLUSTERS FROM THE ROSAT ALL-SKY-SURVEY
}

\author{
MICHAEL J. LEDLOW \\ University of New Mexico, Institute for Astrophysics \\ Dept of Physics \& Astronomy, Albuquerque, NM USA \\ WOLFGANG VOGES \\ Max-Planck-Institut für Extraterrestrische Physik \\ Garching bei München, Germany \\ FRAZER N. OWEN \\ National Radio Astronomy Observatory \\ Socorro, NM USA \\ AND \\ JACK O. BURNS \\ University of Missouri, Office of Research \\ Columbia, MO USA
}

Using the ROSAT All-Sky-Survey (RASS), we examine the X-ray properties of a statistically complete sample of 294 nearby $(z<0.09)$ Abell clusters from our VLA $20 \mathrm{~cm}$ survey (Ledlow \& Owen 1995) and 49 Poor Groups $(z<0.03)$ (Burns et al. 1996). Our analysis includes a catalog of all significant $(>3 \sigma)$ X-ray peaks, an analysis of the X-ray extents, identification of ICM emission, comparison to optical cluster properties, and a cross-correlation with our radio galaxy catalogue. We will make optical/Xray overlays of the cluster fields available over the WWW in the near future (see http://astro.nmsu.edu/ mledlow for updates).

\section{References}

Burns, J.O., et al. 1996, ApJ, 467, L49

Ebeling, H., et al. 1997, ApJ, 479, 101

Ledlow, M.J., \& Owen, F.N. 1995, $A J$, 110,1959 


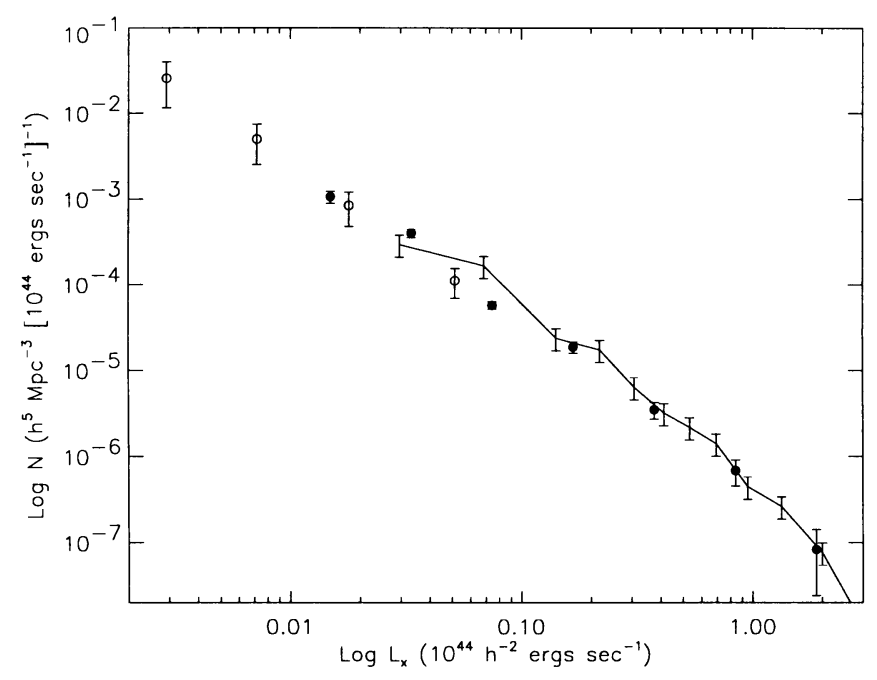

Figure 1. The X-ray luminosity function for our nearby rich clusters sample (solid circles), the poor clusters from Burns et al. (1996) (open circles), and the data points from the Brightest Cluster Sample of Ebeling et al. (1997) - solid line. We use $H_{0}=100 \mathrm{~km} \mathrm{sec}^{-1} \mathrm{Mpc}^{-1}$. 